\title{
Fracture Liaison Service and the Prospect of Fragility Refracture in Osteoporotic Patients
}

\author{
Tarik Wasfie $^{1 *}$, Avery Jackson ${ }^{2}$ and Natalia Cwalina ${ }^{1}$ \\ 1Department of Trauma Surgery, Ascension Genesys Hospital, 1 Genesys Parkway, Grand Blanc, Michigan, USA \\ 2Department of Neurological Surgery, Ascension Genesys Hospital, 1 Genesys Parkway, Grand Blanc, Michigan, USA
}

${ }^{*}$ Corresponding author: Tarik Wasfie, Department of Trauma Surgery, Ascension Genesys Hospital, 1 Genesys Parkway, Grand Blanc, Michigan, USA; Email: Tarik.Wasfie@ascension.org

Received: April 05, 2019; Accepted: April 15, 2019; Published: April 17, 2019;

Osteoporosis is a silent disease, but one who's impact is not silent. Over 9 million Americans have been diagnosed with osteoporosis, and more than 2 million osteoporotic fractures occur per year. This means that one in every two women aged 50 and above will have an osteoporotic fracture in her lifetime. Osteoporosis is characterized by decreased bone strength, reduced bone quantity and a decrease in the bone quality. These three factors lead to an increased susceptibility to fractures.

Postmenopausal women incur a high incidence of osteoporosis and subsequently fragility fractures A (defined as a fracture with minimal or no trauma, that occurs to the spine, ribs, pelvis or extremity bones), with a $50 \%$ refracture rate within two years. Men are not immune from this as $30 \%$ of men over 50 will have it on average. The burden on society and cost are high (around 21 million dollars in 2006) and will only grow as the aging population increases worldwide. Measures to prevent and reduce refracture rates and thus readmission have been worked out.

One of these ideas is a Fracture Liaison Service (FLS). The FLS program was established by the National Osteoporosis Foundation in 1996. It is a coordinated preventative care model that is operated under the supervision of a bone health specialist, who also collaborates with the patient's primary care physician.

The Fracture Liaison Service project follows patients after a fragility fracture occurs. These patients are examined in a clinic, undergoing multiple tests including serum calcium, vitamin D levels as well as a dual-energy X-ray absorptiometry (DEXA scan). Their fracture risk is assessed following multiple visits at regular intervals to evaluate any progression of disease and to determine the refracture rate.

At our hospital, we established a Fragility Liaison Service program which remains solely the responsibility of the treating surgeon. The clinic since its inception in 2015 has seen around 300 patients and significantly reduced refracture rates. We focused primarily on patients with fractures of the spine and were able to reduce refracture rates by $1 / 3$ in all patients with refractures (56\% without FLS vs $37 \%$ post FLS, $\mathrm{p}=0.01$ ).

However, our clinic can only see a certain number of patients with vertebral fractures, thus the remaining patients with rib fractures, pelvic fractures and extremity fractures need to be addressed. This can only happen when third-party payers pay attention to the FLS program as it has proven beneficial. 